\title{
Biology of Renal Tubulo-Interstitial Cells
}

\author{
Marc E. De Broe Mark J.F. Helbert \\ Department of Nephrology, University of Antwerp, Belgium
}

In the last 10 years there has been an exponential increase in the number of scientific reports on the methodological and mechanical aspects of renal tubular cell culture. During 2 days, a number of investigators from leading laboratories studying renal tubular cell cultures convened and discussed their latest results in Antwerp.

In a first session dealing with the current methodological aspects in renal primary cell practice, two pioneers of renal cell culture, D.E. Sens (Morgantown, W.Va., USA) and A. Trifillis (Baltimore, Md., USA), presented an overview of the methodology used to obtain human proximal tubular cells and collecting duct cells.

Clemans Grupp and colleagues (Göttingen, Germany) discussed the complex issue of interstitial cells of the kidney and presented different techniques for the isolation of the fibroblasts, and their identification in cultures by morphological criteria since a specific marker for the different fibroblasts is still not available. The recent working hypothesis of the transformation of a fibroblast towards myofibroblast was discussed.

One of us (M.H., Antwerp, Belgium), demonstrated that flow sorting of cells prior to culture is a straight forward way to obtain pure cultures of cells from defined nephron segments, using specific markers. Cell populations sorted with these markers were shown to retain the

\begin{tabular}{ll}
\hline KARGER & (1) 1999 S. Karger AG, Basel \\
Fax +4161306 1234 & 1018-7782/99/0076-0341\$17.50/0 \\
$\begin{array}{l}\text { E-Mail karger@karger.ch } \\
\text { www.karger.com }\end{array}$ & $\begin{array}{l}\text { Accessible online at: } \\
\text { www.karger.com/journals/exn }\end{array}$
\end{tabular}

functional characteristics of their in vivo counterparts. By applying these validated markers on cultures, starting from a mixture of cells, it was shown that (1) the different cell types indeed remain identifiable flowcytometrically in short-term cultures, and (2) the presence of two or more identifiable cell types in these cultures allows comparison of responses by distinct distal cell types simultaneously in a single culture using multiparametric flowcytometry.

A. Vandewalle (Paris, France) showed that targeted oncogenesis in transgenic mice, where an oncogene is placed under the control of the regulatory sequences of a cell-specific gene, has been used to derive several new types of differentiated nonepithelial and epithelial cell lines. He summarized the properties of cell lines derived from the proximal, distal and collecting duct cells.

Will Minuth (Regensburg, Germany) has used an in vitro model for many years to investigate the terminal differentiation mechanisms leading from embryonic to adult renal tissue. A special type of container allows continuous superfusion of the epithelia with individual media on the luminal and basal side. By this method it becomes possible to culture embryonic collecting duct epithelial in a fluid gradient for weeks. They demonstrated nicely that differentiation is not always under the control of growth factors but is also regulated by the electrolyte environment.
Marc E. De Broe, MD, PhD

Department of Nephrology-Hypertension, University of Antwerp p/a University Hospital Antwerp, Wilrijkstraat 10

B-2650 Edegem/Antwerpen (Belgium)

Tel. +32 38213421, Fax +32 3829 0100, http://nefro-www.uia.ac.be/nefro/ 
Gérard Friedlander (Paris, France) demonstrated the advantages and possible drawbacks of cultured cells in the elucidation of phenotypic changes induced by transgenesis. They also pointed out the need to refer to results obtained in vivo to validate in vitro models on a molecular basis.

Antisense oligodeoxynucleotides offer the potential to block the expression of specific genes with the goal of altering the phenotypic behavior of the cells. Michael B. Ganz (Cleveland, Ohio, USA) demonstrated that this technique was recently used for in vitro and in vivo studies in renal cells. The use of antisense technology has been applied in vitro to help define both the normal mechanisms of specific ion transport and function and the pathobiological processes leading to glomerular proliferation and matrix formation.

The role of the proximal tubular cell as mediator of interstitial inflammation and tissue injury/fibrosis was demonstrated by Carla Zoja (Bergamo, Italy) who presented several lines of direct and indirect evidence that glomerular capillary hypertension leads to impaired glomerular permselectivity to proteins, which are then filtered in excessive amounts and accumulate in the lumen of the proximal tubules. The secondary process of tubular epithelial endocytosis of filtered protein may contribute substantially to promote an interstitial inflammatory reaction that consistently evolves to renal scarring. She has given arguments to show that overloading proximal tubular cells with various proteins results in a common pathway of activation of NF- $\mathrm{KB}-$ dependent genes whose protein products may possibly play a determinant role in recruiting inflammatory cells into renal interstitium. Treatment of rats with an ACE inhibitor while reducing proteinuria also suppressed $\mathrm{NF}-\kappa \mathrm{B}$ activation, reduced renal MCP-1 and mononuclear cell infiltrates.

Cees van Kooten (Leiden, The Netherlands) summarized the potential role of renal tubular epithelial cells in the production of early inflammatory mediators like complement, cytokines and growth factors. He demonstrated that there is still little insight into the various stimuli involved in the activation of these cells in the different signal transduction pathways and in the fine tuning by the different cytokines. He underscored the importance that there is preferential expression of receptors or selective secretion of cytokines at the apical or basolateral side of these cells. He emphasized the importance of the expression or proximal tubular epithelial cells of a wide variety of surface peptidases including CD10, CD13, CD26 and CD143. He underscored the importance of CD143 since it can generate active angiotensin II and showed that CD26 can truncate the N-terminal part from RANTES, thereby changing its specificity, suggesting a new level of complexity in the regulation of inflammatory tubular epithelial cells.

James McLay (Aberdeen, Scotland) demonstrated that primary cultures of human proximal tubular cells can be stimulated by a combination of pro-inflammatory cytokines to produce high levels of nitric oxide (NO). They have demonstrated the possible immunomodulatory interaction between the natriuretic peptides and $\mathrm{NO}$ in the proximal tubule. The ANF/NO interaction places ANF in the unique position of being able to maintain the essential or protective actions of $\mathrm{NO}$ while inhibiting potentially cytotoxic or detrimental effects associated with high levels of NO.

The data presented by Aled Phillips (Cardiff, Wales) demonstrated that the glucose-induced accumulation of type-IV collagen and fibronectin was associated with alterations in the degradative pathways of these matrix components. In addition, fibronectin generation in response to glucose was nonpolar in terms of application of glucose, but polar in terms of fibronectin accumulation. The mechanisms of glucose-induced modulation of fibronectin were mediated by polyol pathway activation, and more specifically related to the metabolism of sorbitol to fructose.

Jill T. Norman (London, UK) showed that hypoxia is a potent fibrogenic stimulus for human renal tubular epithelial cells and interstitial fibroblast in vitro, increasing ECM (collagen) production and decreasing matrix turnover via effects on degradative enzymes and/or on their inhibitors and acting through TGF $\beta 1$-independent pathways. The hypoxia-induced increase in TIMP-1 and coll-I is dependent on de novo gene transcription and protein synthesis. In renal cells the oxygen sensor is likely to be a heme protein similar to that postulated in other cell types and the hypoxia-induced fibrogenic response in renal fibroblasts involves activation of both protein kinase Cand tyrosine kinase-mediated signal transduction pathways.

Finally Heather J. Saunders (Sydney, Australia) discussed the value of human renal tubular cells in the study of mechanisms of human tubulointerstitial pathology. They used cyclosporine as an in vitro model of tubular interstitial disease and demonstrated that clinically relevant concentrations are directly toxic to human tubulointerstitial cells, promote interstitial extracellular matrix accumulation by a combination of suppressed matrix degradative enzyme activity, enhance cortical fibroblast 
synthesis via induction of autocrine IGF-I secretion and action, and increase proximal tubular cell secretion of TGF $\beta_{1}$. The finding that modulation of local cytokine networks plays a key role in this in vitro model of tubulointerstitial disease raises the possibility that more specific targeting of therapies to reduce fibrogenic cytokine activity within the cortical tubulointerstitium may improve our ability to prevent progressive tubulointerstitial disease following nephrotoxic injury.

Primary cultures of human renal cells are suited to the performance of future studies for the therapeutic efficacy of gene therpay, decorin, growth factor receptor antagonist and somatostatin analogues. They will greatly enhance our understanding of the molecular mechanisms, and ultimately, treatment of progressive tubulointerstitial disease.

\section{Acknowledgement}

The authors wish to thank the Fund of Scientific Research (FWO), University of Antwerp, and Zeneca Belgium for the support which made this publication possible. 